\title{
Determining intrafractional prostate motion using four dimensional ultrasound system
}

\author{
Mariwan Baker ${ }^{1,2,3^{*}}$ and Claus F. Behrens ${ }^{1}$
}

\begin{abstract}
Background: In prostate radiotherapy, it is essential that the prostate position is within the planned volume during the treatment delivery. The aim of this study is to investigate whether intrafractional motion of the prostate is of clinical consequence, using a novel 4D autoscan ultrasound probe.

Methods: Ten prostate patients were ultrasound (US) scanned at the time of CT imaging and once a week during their course of radiotherapy treatment in an ethics-approved study, using the transperineal Clarity autoscan system (Clarity ${ }^{\oplus}$, Elekta Inc., Stockholm, Sweden). At each US scanning session (fraction) the prostate was monitored for 2 to 2.5 min, a typical beam-on time to deliver a RapidArc ${ }^{\oplus}$ radiotherapy fraction. The patients were instructed to remain motionless in supine position throughout the US scans. They were also requested to comply with a bladder-filling protocol. In total, 51 monitoring curves were acquired. Data of the prostate motion in three orthogonal directions were analyzed. Finally, the BMI value was calculated to investigate correlation between BMI and the extent of prostate displacement.
\end{abstract}

Results: The patients were cooperative, despite extra time for applying the TPUS scan. The mean ( $\pm 1 \mathrm{SD})$ of the maximal intrafractional displacements were $[\mathrm{mm}] ; \mathrm{I}(+) / \mathrm{S}:(0.2 \pm 0.9) ; \mathrm{L}(+) / \mathrm{R}:(-0.2 \pm 0.8)$; and $\mathrm{A}(+) / \mathrm{P}:(-0.2 \pm 1.1)$, respectively. The largest displacement was $2.8 \mathrm{~mm}$ in the posterior direction. The percentage of fractions with displacements larger than $2.0 \mathrm{~mm}$ was $4 \%, 2 \%$, and $10 \%$ in the IS, LR, and AP directions, respectively. The mean of the maximal intrafractional Euclidean distance (3D vector) was $0.9 \pm 0.6 \mathrm{~mm}$. For $12 \%$ of the fractions the maximal 3D vector displacements were larger than $2.0 \mathrm{~mm}$. At only two fractions (4 \%) displacements larger than 3. $0 \mathrm{~mm}$ were observed. There was no correlation between BMl and the extent of the prostate displacement.

Conclusions: The prostate intrafractional displacement is of no clinically consequence for treatment times in the order of $2-2.5$ min, which is typical for a RapidArc radiotherapy fraction. However, prostate motion should be considered for longer treatment times eg if applying conventional or IMRT radiotherapy.

\section{Background}

In external beam radiotherapy of the prostate, it is essential that the prescribed dose is precisely delivered to the prostate, while reducing toxicity to rectum and bladder. This can be achieved by daily accurate positional verification of the prostate using image-guided radiation therapy (IGRT) $[1,2]$. Since variation in prostate position during treatment delivery (intrafractional prostate motion) might occur, the target may be underdosed due to a

\footnotetext{
*Correspondence: mariwan.baker@regionh.dk

'Department of Oncology, Radiotherapy Research Unit, Herlev Hospital, University of Copenhagen, Herlev, Denmark

${ }^{2}$ Center for Fast Ultrasound Imaging, Department of Electrical Engineering,

Technical University of Denmark, DK-2800 Lyngby, Denmark

Full list of author information is available at the end of the article
}

possible prostate shift $[3,4]$. Bladder filling variations, rectal volume changes, and respiratory motion are some of the factors that might lead to intrafractional prostate motion [5-7].

To determine the intrafractional prostate motion, various imaging techniques have been investigated in different studies, such as X-ray imaging, kilovoltage $(\mathrm{kV})$ 3D CBCT and megavoltage (MV) imaging, Cine-MRI, in-room $\mathrm{CT}$, implanted markers and transponders, and ultrasound [8-11]. Most of the studies are based on acquiring pre- and post-treatment images $[12,13]$.

(c) 2016 The Author(s). Open Access This article is distributed under the terms of the Creative Commons Attribution 4.0 International License (http://creativecommons.org/licenses/by/4.0/), which permits unrestricted use, distribution, and reproduction in any medium, provided you give appropriate credit to the original author(s) and the source, provide a link to the Creative Commons license, and indicate if changes were made. The Creative Commons Public Domain Dedication waiver (http://creativecommons.org/publicdomain/zero/1.0/) applies to the data made available in this article, unless otherwise stated. 
Various techniques are developed to enable real-time online prostate localization and monitoring, such as: tracking implanted electromagnetic transponders (Calypso Medical Technologies, Seattle, WA), and tracking fiducial markers (FMs) or implanted radioactive seeds (in Brachytherapy) using real-time X-ray imaging. Recently, transperineal ultrasound (TPUS) autoscan (Clarity ${ }^{\oplus}$, Elekta Inc., Stockholm, Sweden), a non-ionizing, non-invasive imaging modality, has been developed to allow real-time prostate tracking [14]. Various studies have investigated the Calypso system to determine intrafractional prostate motion [15-19], however, to our knowledge, there is only one study (Ballhausen et al.) using the Clarity TPUS system [20]. In the study by Ballhausen et al. data from 6 prostate patients are investigated. Ballhausen et al. concludes that "intrafraction motion of the prostate is a random walk" and the prostate moves away from the isocenter during treatment delivery. In the present study, the TPUS system was utilized to determine the intrafractional motion of the prostate.

Using advanced radiotherapy techniques, such as volumetric modulated arc therapy (VMAT) or RapidArc ${ }^{\oplus}$ Radiotherapy Technology, the treatment delivery time can be significantly reduced, thus minimizing intrafractional prostate motion [21, 22]. In our institution, RapidArc radiotherapy is a standard technique for treating prostate cancer patients, and a typical beam-on time for a treatment fraction is approximately $2.5 \mathrm{~min}$.

The aim of this study is to investigate whether the intrafractional prostate motion, during a time interval corresponding to the beam-on time for RapidArc, is within $2.0 \mathrm{~mm} .2 \mathrm{~mm}$ is a tolerance value which is perceived to be clinically irrelevant according to the British Ionization Radiation Medical Exposure Regulations 2000 (IRMER 2000).

\section{Methods \\ Patients}

Ten prostate cancer patients, with an average age of 68 years (range $58-76$ years), were US scanned in the CT room and once a week in the treatment room utilizing the Clarity 4D TPUS monitoring system. All post prostatectomy patients were excluded. The patients received a cumulative dose of $76 \mathrm{~Gy}$ in 38 fractions, 2.0 Gy per fraction, 5 fractions per week. Table 1 . comprises patient specifics, US scans, and the treatment technique. The TPUS scans were performed by six radiation therapy technologists (RTTs). The study was approved by the Danish national ethical committee, and voluntary informed consent was obtained for each participant according to the World Medical Association Declaration of Helsinki $(1975 / 2000)$. The patients were placed in a supine position and instructed to remain motionless throughout the subsequent scans. To ensure better image quality, the patients
Table 1 Patients characteristics, ultrasound scans, and treatment technique

\begin{tabular}{ll}
\hline Age $(Y)$ & 68 \\
Mean & $58-76$ \\
Range & \\
BMI & 27 \\
Mean & $22-37$ \\
Range & \\
PSA level, ng/mL & 12.4 \\
Mean & $2-22$ \\
Range & \\
Differentiation (Gleason Score) & 5.7 \\
Mean & $2.4-8.8$ \\
Range & \\
Fractions & 38 \\
Total fractions & 2.0 \\
Dose/fraction [Gy] & Danish patients \\
Race/ ethnicity & Excluding all post prostatectomy \\
Prostate patients & Only external radiotherapy (RapidArc) \\
Radiation treatment & A standard bladder filling protocol \\
Bladder filling & $4-6$ scans \\
Number of US scans/patient & 51 scans \\
Total number of US scans & \\
\hline
\end{tabular}

were requested to comply with a moderate bladder-filling protocol.

\section{Clarity ultrasound system}

The Clarity system consists of two mobile units (one in the $\mathrm{CT}$ room and the second in the treatment room), which are connected through a workstation/server. The workstation was used for target delineation and retrieving the prostate monitoring curves. Details of US 3D image reconstructions and the precision of the system are explained thoroughly in a previous study [23]. In short, a ceiling-mounted infrared (IR) camera recognizes the US probe by detecting the IR-reflectors affixed to them. This is essential for determining the geographical position of the reconstructed anatomical structures.

To enable superimposition of the reconstructed US images from the 4D autoscan probe on the CT images, the system was calibrated to the same coordinate system as the CT simulation and treatment rooms. The calibration procedure was accomplished by means of a dedicated alignment phantom provided by the vendor.

\section{CT room}

All patients underwent a treatment planning $\mathrm{CT}$ scan followed by an MRI scan. After the CT-scan the 3D-TPUS scan was acquired. The patient was instructed to remain 
still during image acquisition and US monitoring. Afterwards, the US-CT fusion was performed, on which the prostate volume was delineated and used as a reference for weekly US imaging in the treatment room.

\section{Autoscan probe}

The autoscan-US probe consists of a one dimensional (1D) transducer array of 128 elements, using a central frequency of $5.0 \mathrm{MHz}$. The probe is provided with IR reflectors fixed in a way that can be detected by the ceiling mounted IR-camera. Initially, the probe is affixed to a TPUS kit, placed over the coach under the patients' knees, and pushed gently to the patient for scanning. The motorized head is capable of real-time online scanning of the prostate.

\section{Prostate monitoring in the treatment room}

The patient was prepared for daily treatment by aligning him to the lasers guided by reference marks (tattoos) on the skin, ie to reproduce the patient's setup position from the CT-simulation room. Prior to treatment, the inter-fraction patient positioning was corrected for by daily $\mathrm{kV}$ images by utilizing three implanted FMs. Once a week and immediately after treatment delivery the TPUS monitoring system was set up for real-time tracking of the prostate. The time dependent prostate displacements (prostate intrafractional motion) were recorded in 2 to $2.5 \mathrm{~min}$. For each patient, 4-6 US scans were acquired. The prostate displacements in the three orthogonal directions were recorded for retrospective analysis.

\section{Statistical analysis}

The prostate data, showing real-time prostate $\mathrm{COM}$ position in the Inferior-Superior (I/S), Left-Right (L/R) and Anterior-Posterior $(\mathrm{A} / \mathrm{P})$ directions, were evaluated using the Clarity workstation by one observer (MB). For some fractions, irregular fluctuating data at the beginning of the monitoring curve were discarded. The maximum prostate displacement in each direction was recorded for each fraction. Furthermore, the Euclidean distance (3D vector) was computed and the maximal $3 \mathrm{D}$ vector was recorded for each fraction. Finally, the mean $( \pm 1$ standard deviation (SD)) of the maximal displacements in each direction and for the $3 \mathrm{D}$ vector were calculated. The BMI value for each patient was calculated to investigate correlation between magnitudes of the displacement against the BMI. For the data and statistical analysis the statistical program R (version 2.15.3) was used.

\section{Results}

All the patients enrolled in this study were cooperative, despite the extra time of about $10 \mathrm{~min}$ for each fraction needed for the autoscan setup and prostate monitoring.
None of the patients expressed any discomfort during the autoscan setup, nor from the TPUS real-time scanning.

Data analysis of the 51 TPUS scans resulted in mean values $( \pm 1 \mathrm{SD})$ of the maximal intrafractional displacements of the prostate $[\mathrm{mm}] ; \mathrm{I}(+) / \mathrm{S}:(0.2 \pm 0.9) ; \mathrm{L}(+) / \mathrm{R}$ : $(-0.2 \pm 0.8)$; and $\mathrm{A}(+) / \mathrm{P}:(-0.2 \pm 1.1)$. The largest displacement was $2.8 \mathrm{~mm}$ in the posterior direction. The percentage of fractions larger than $2.0 \mathrm{~mm}$ was $4 \%, 2 \%$, and $10 \%$ in the IS, LR, and AP directions, respectively. The mean Euclidean distance of the 51 scans was $0.9 \pm$ $0.6 \mathrm{~mm}$. The percentage of fractions with $3 \mathrm{D}$ vector displacement larger than $2.0 \mathrm{~mm}$ was $12 \%$, and only 2 scans $(4 \%)$ showed displacements larger than $3.0 \mathrm{~mm}$. No correlation was found between BMI-value and the magnitude of the prostate displacement.

The monitoring curves of the prostate show variations in prostate displacement between different fractions (Fig. 1). A common zero point, start point for the curves, was established by choosing an arbitrary position-value for each scan/curve, a position immediately after the prostate tracking initiated, and then subtracting that value from the rest. For some fractions, the tendency of larger intrafractional displacement for prolonged observation times can be observed. The figure also indicates that the extent of the prostate intrafractional displacement has its largest dispersal in the $\mathrm{A} / \mathrm{P}$ direction, and least displacements in the $\mathrm{L} / \mathrm{R}$ direction.

Figure 2a-c presents boxplots of the maximal displacements at each fraction for each of the ten patients in $\mathrm{I} / \mathrm{S}$, $\mathrm{L} / \mathrm{R}$, and $\mathrm{A} / \mathrm{P}$ directions, respectively. The horizontal band inside the box indicates the second quartile (median), the lower and the upper edges of the box indicate the first $\left(25^{\text {th }}\right)$ and third $\left(75^{\text {th }}\right)$ quartiles. Furthermore, the lower and the upper extremes of the whiskers display the minimum and maximum values in the absence of single data point outliers. The individual boxplot of maximal prostate displacements is based on analysis of 4-6 TPUS scans per patient. As can be observed, there are some variations in prostate displacements between the patients and in the different directions. The overall maximal prostate displacement in all three directions and the Euclidean 3D vector were shown to be less than $2.0 \mathrm{~mm}$ for most of the fractions (Fig. 2d).

\section{Discussion}

Intrafractional prostate motion has been investigated previously using pre- and post-treatment imaging. One weakness in applying this method is inter-observer matching uncertainty [24]. Despite matching variability, using portal imaging of seeds, there is an additional error associated with the thickness of the CT-slices that may introduce further uncertainty into the determination of the displacement. Another drawback is that the prostate can be displaced and then revert to its initial 


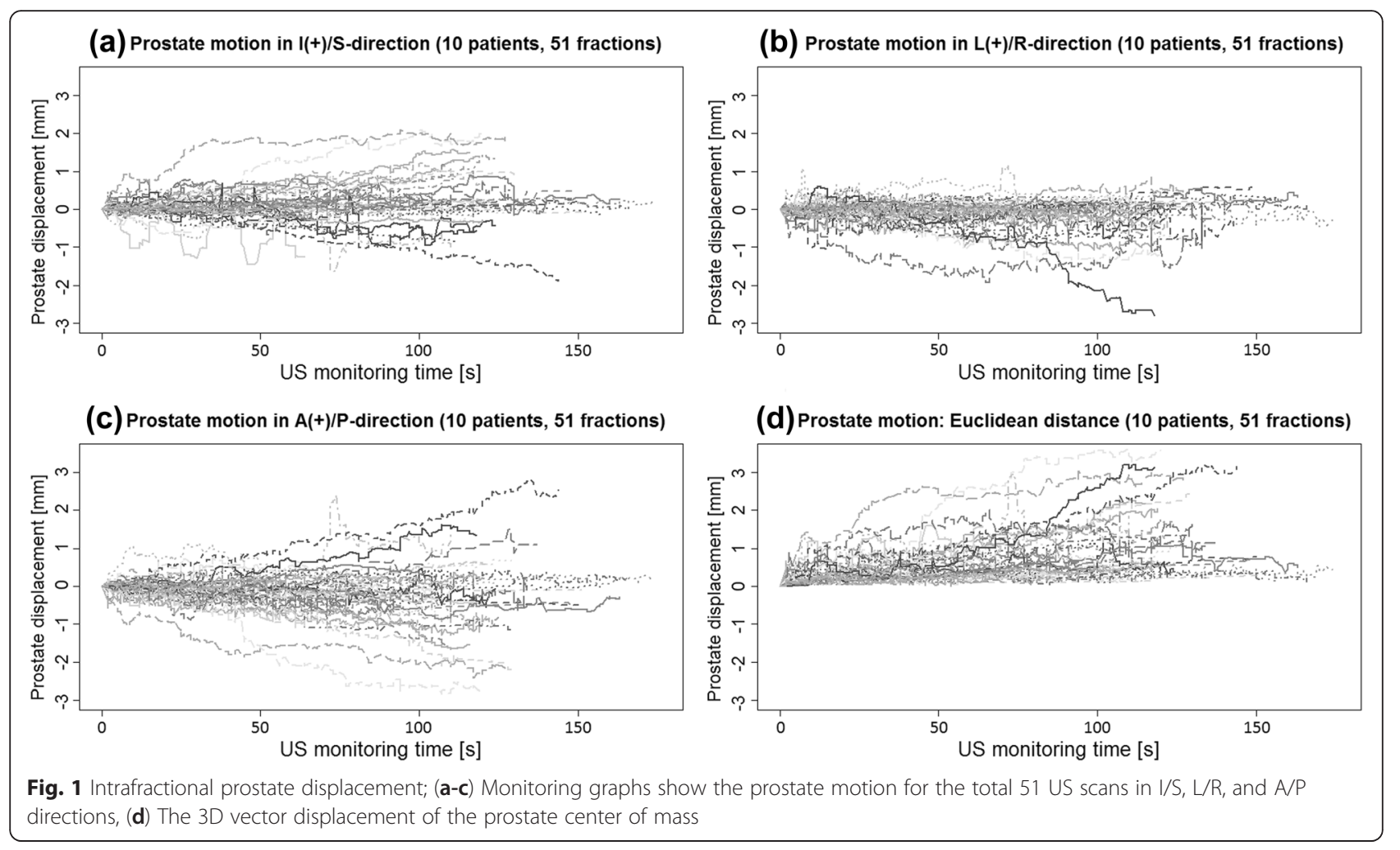

(a) Intra-fractional prostate displacements per patient in $\mathrm{I}(+) / \mathrm{S}$ direction

(b) Intra-fractional prostate displacements per patient in $L(+) / R$ direction
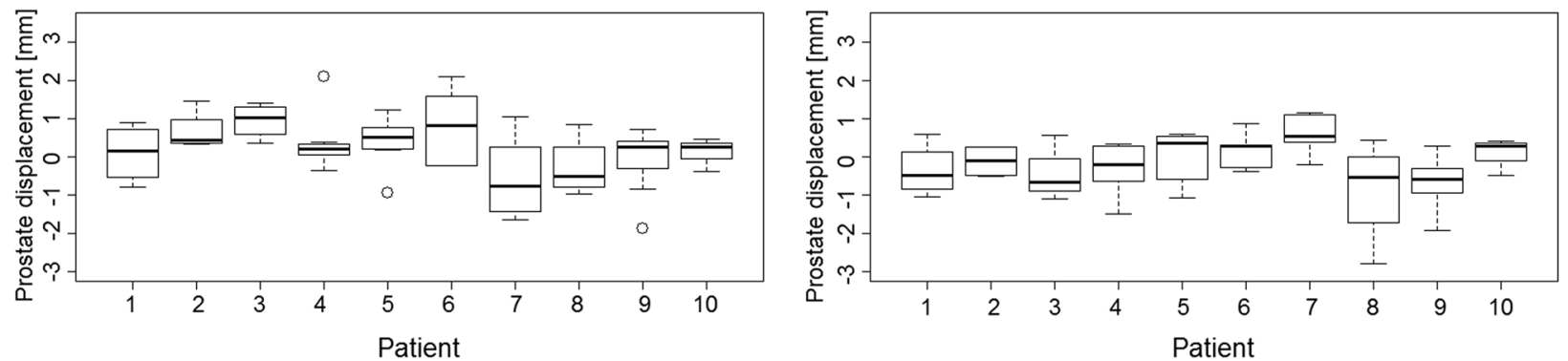

(c) Intra-fractional prostate displacements per patient in A(+)/P direction

(d) Overall prostate shifts in 3 directions and Euclidean (51 fractions)
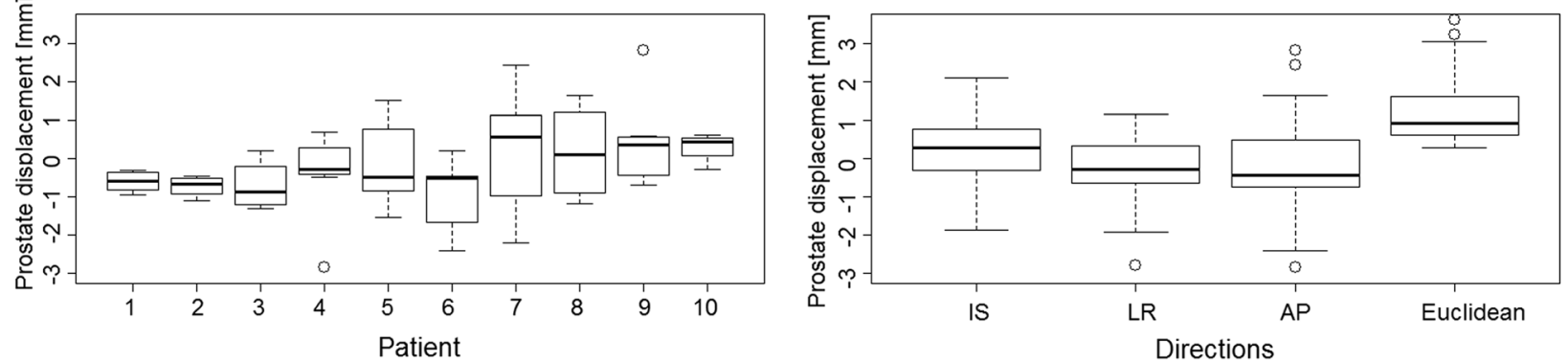

Fig. 2 Boxplots of the intrafractional prostate displacements; (a-c) Boxplots of the prostate displacements for ten patients in I/S, L/R, and A/P directions. (d) Boxplot of overall prostate displacements in I/S, L/R, and A/P directions, including Euclidean (3D vector) distance 
position, as can be observed in Fig. 1, which cannot be detected by only a single snapshot pre- and posttreatment image [25]. Therefore real-time tracking is a proper monitoring method to accurately detect intrafractional prostate motion. Table 2 tabulates the results of the present study and previously reported data.

In this study the 4D TPUS monitoring system was shown to be able to track the prostate. The $4 \mathrm{D}$ autoscan probe have previously been applied as a reliable tool to measure the prostate displacement during transabdominal probe simulation [26]. The operators, with experience from frequently used transabdominal scanning, reported that using the TPUS system, it was easier to acquire images and identify soft tissue structures compared to transabdominal scanning. In a previous phantom study, Abramowitz et al. found good agreement between the TPUS autoscan and Calypso system (Varian Medical Systems, Palo Alto, CA) in tracking the embedded prostate-like sphere [27]. The Calypso system has been investigated in different studies, which have confirmed that it is an accurate monitoring method for tracking the prostate gland.

In the current study, prostate monitoring was limited to approximately $2.5 \mathrm{~min}$, a typical beam-on time to deliver a RapidArc fraction. We observed that the prostate is not stationary during the tracking time, and displacements tend to increases with the elapsed monitoring time, which is in line with the findings of other published papers [20]. Ballhausen et al. found that the intrafractional motion of the prostate tends to "a linear increase of the variance" with the duration of the fraction. Furthermore, we found that the displacement varies for different fractions and also for different patients.

In our study, we noticed that, for most of the fractions, the intrafractional prostate motion was mostly smaller than $2 \mathrm{~mm}$, for a period of 2 to $2.5 \mathrm{~min}$. Comparably, Choi et al. [28], using transrectal ultrasound scans to track three implanted fiducial markers (12 patients and 336 fractions), observed that only $11 \%$ of the $3 \mathrm{D}$ vector displacements were larger than $2 \mathrm{~mm}$ during 4-5 min of monitoring (Table 2). Similarly, Li et al. [4], utilizing a Calypso tracking system (35 patients and 1267 fractions), reported that the intrafractional motion of the prostate is mostly less than $2 \mathrm{~mm}$, especially for the first three minutes of tracking. They concluded that a CTV-PTV margin of $2 \mathrm{~mm}$ is adequate to ensure covering the target with the planned prescribed dose, despite larger intrafractional uncertainty for some of the patients. Furthermore, Vargas et al. [29], in an Cine-MRI study, calculated the CTV-PTV margin to be $2.1 \mathrm{~mm}$, comparable to Li et al.'s, which would account for the intrafractional motion. They showed also that the prostate motion is smaller in supine than prone patient

Table 2 Intrafraction prostate motion in three directions and 3D vector; a comparison of the present study with previously published data using different systems

\begin{tabular}{|c|c|c|c|c|c|c|c|c|}
\hline \multirow[b]{2}{*}{ Investigator, year } & \multirow[b]{2}{*}{$\mathrm{N}$ (fractions) } & \multirow[b]{2}{*}{ System } & \multirow[b]{2}{*}{ Time $[\mathrm{min}]$} & \multicolumn{3}{|c|}{$\begin{array}{l}\text { Mean of max prostate shifts } \\
( \pm 1 \mathrm{SD})[\mathrm{mm}]\end{array}$} & \multirow[b]{2}{*}{$\% 3 \mathrm{D}$ vector shift } & \multirow[b]{2}{*}{ 3D-vector $[\mathrm{mm}]$} \\
\hline & & & & $\mathrm{I}(+) / \mathrm{S}$ & $L(+) / R$ & $A(+) / P$ & & \\
\hline Lometti et al., 2005 [33] & $11(133)$ & $\begin{array}{l}\text { MV fluoroscopy: real-time FM } \\
\text { tracking }\end{array}$ & 1 & & & & $>2 \mathrm{~mm} 4 \%$ & $0.7 \pm 0.5$ \\
\hline Li HS et al., 2008 [4] & $35(1267)$ & Calypso & 12 & $0.3 \pm 0.7$ & $0.0 \pm 0.3$ & $0.4 \pm 0.6$ & & \\
\hline Kupelian et al., 2008 [2] & $17(550)$ & Calypso & 10 & & & & $>3$ mm $14 \%$ & \\
\hline Langen et al., 2008 [18] & $17(550)$ & Calypso & 2 & & & & $>3$ mm $3 \%$ & \\
\hline Li et al., 2009 [19] & $20(157)$ & Calypso & 11.4 & & & & $>3$ mm $19 \%$ & \\
\hline Vargas et al., 2010 [29] & $7(68)$ & Cine-MRl & 4 & & & & & $0.41 \pm 1.2$ \\
\hline Wang et al., 2011 [34] & $29(1061)$ & Calypso & 3 & & & & $>3$ mm $9 \%$ & \\
\hline $\begin{array}{l}\text { Smeenk et al., } 2011 \\
{[30]}\end{array}$ & $15(576)$ & Calypso & 2.5 & & & & $>3 \mathrm{~mm} 1.4 \%$ & \\
\hline Ng et al., 2012 [32] & $10(268)$ & $\begin{array}{l}\text { Real-time kilovoltage FM } \\
\text { tracking }\end{array}$ & $3-4$ & & & & $>3$ mm $5.6 \%$ & \\
\hline Mayyas et al., 2013 [9] & 8 & $\begin{array}{l}\text { Pre-post-treatment kV } \\
\text { images }\end{array}$ & & $0.8 \pm 2.7$ & $0.2 . \pm 2.1$ & $-0.3 \pm 2.4$ & & \\
\hline Mayyas et al., 2013 [9] & 19 & Calypso & & $0.0 \pm 1.5$ & $0.0 \pm 0.6$ & $0.0 \pm 1.3$ & & \\
\hline Tong et al., 2015 [8] & $236(8660)$ & Calypso & 2 & & & & $>2$ mm $13 \%$ & \\
\hline Choi et al., 2015 [28] & $12(336)$ & Transrectal US/3 FMs & $4-5$ & $0.6 \pm 0.6$ & $-0.3 \pm 0.3$ & $-0.7 \pm 0.6$ & $>2$ mm $11 \%$ & $1.1 \pm 0.8$ \\
\hline Present study & $10(51)$ & Clarity real-time TPUS & $2-2.5$ & $0.2 \pm 0.9$ & $-0.2 \pm 0.8$ & $-0.2 \pm 1.1$ & $>2$ mm $12 \%$ & $0.9 \pm 0.6$ \\
\hline
\end{tabular}

Intrafractional motion of prostate patients $(\mathrm{N})$ in 3D vector, $\mathrm{I} / \mathrm{S}$, inferior-superior, $\mathrm{L} / \mathrm{R}$, left-right, and $\mathrm{A} / \mathrm{P}$, anterior-posterior directions, using TPUS, transperineal Clarity ultrasound (US) system, Calypso system, implanted fiducial markers (FMs), CINE-MRI, and endorectal balloon 
positioning. On the other hand, Smeenk et al. [30], again using Calypso system (15 patients and 576 fractions), discovered that prostate motion in $3 \mathrm{D}$ vector was larger than $3 \mathrm{~mm}$ in only $1.4 \%$ of the fractions during the first $2.5 \mathrm{~min}$, but increased to $18 \%$ for a monitoring duration of $10 \mathrm{~min}$. Comparably, Tong et al. [8], also using Calypso tracking system (236 patients and 8660 fractions), showed that up to $20 \%$ of the prostate shift can be larger than $3 \mathrm{~mm}$ when tracking time is $12 \mathrm{~min}$. But they ascertained that the prostate motion, for the first three minutes, is very small $(<2 \mathrm{~mm})$ for most patients. Similarly, Shelton et al. [31] investigated the intrafraction prostate motion, once again using Calypso system (37 patients and 1332 fractions), and found that the overall mean value of the $3 \mathrm{D}$ vector displacement is less than $3 \mathrm{~mm}$ for the first $3 \mathrm{~min}$, but increased with duration time to up to $6.5 \mathrm{~mm}$ in the fourteenth minute. Likewise, Kupelian et al. [2], once more using Calypso system (17 patients and 550 fractions) detected that during $10 \mathrm{~min}$ monitoring in $14 \%$ of the fractions the shift was larger than $3 \mathrm{~mm}$. Furthermore, $\mathrm{Ng}$ et al. [32], in a study of real-time FM tracking, observed that in $62 \%$ of the fractions (10 patients, 268 fractions) the $3 \mathrm{D}$ vector displacement of the prostate, during 3-4 min tracking was smaller than $1 \mathrm{~mm}$, and corresponded values were $95 \%, 82 \%$, and $80 \%$ in the LR, IS, and AP directions, respectively. They discovered that only $6 \%$ of the shifts were larger than $3 \mathrm{~mm}$. Moreover, Langen et al. [18], as well using Calypso system (17 patients and 550 fractions), spotted that only $3 \%$ of the shift is larger than $3 \mathrm{~mm}$ while tracking the prostate for about 2 min. In addition, Lometti et al. [33], using MV fluoroscopy; real-time FM tracking (11 patients and 133 fractions), confirmed that in only $4 \%$ of the fractions the displacement is larger than $2 \mathrm{~mm}$ while tracking for only one minute. Last, Mayyas et al. [9], in a comparison study of four different systems (27 patients and 1100 fractions), found that the percentage of intrafractional shifts within $\pm 1.5 \mathrm{~mm}$ was $76 \%, 94 \%$, and $82 \%$ in the I/S, L/R, and A/P directions. In summary, the published evidence in combination with this study justifies the assertion that the intrafractional motion of the prostate is insignificant during the first $2.5 \mathrm{~min}$, thus not of clinical consequence while employing advanced VMAT/ RapidArc.

As all other studies, there are limiting factors in the current study; Firstly, the study does not reveal the magnitude of the intrafractional prostate rotation or form deformation. Second, the number of patients and fractions are limited. Third, a dosimetric study is necessary to investigate the impact of the larger prostate displacements on the clinical treatment outcome of the treatment.

\section{Conclusion}

Intrafractional prostate displacement varies between fractions and for different patients. The displacement is insignificant for the first $2-2.5 \mathrm{~min}$ of the monitoring time, but increases with elapsed time. Consequently, prostate shifts during conventional and IMRT treatment delivery should be taken into consideration, but are clinically inconsequential while employing advanced VMAT/ RapidArc techniques.

\begin{abstract}
Abbreviations
3D, Three dimensional; 4D, Four dimensional; A/P, Anterior-posterior; CT, Computed tomography; CTV, Clinical target volume; FM, Fiducial marker; I/S, Inferior-superior; IGRT, Image-guided radiation therapy; IMRT, Intensity modulated radiation therapy; kV, Kilo-voltage; L/R, Left-right; $\mathrm{MHz}$, Mega hertz; MRI, Magnetic resonance imaging; MV, Mega-voltage; PTV, Planning target volume; RTT, Radiation therapy technologist; SD, Standard deviations; TPUS, Transperineal ultrasound; US, Ultrasound; VMAT, Volumetric modulated arc therapy
\end{abstract}

\section{Acknowledgements}

We would like to thank all our colleagues who helped to conduct this study; Gullander L. (RTT), Pedersen S. K (RTT), Zarp T (RTT), Jacobsson S (RTT),

Pazhang S (radiographer) and Lynnerup V. K (radiographer). We would also like to thank Elekta Ltd for their support.

Funding

Elekta, Stockholm, Sweden, has provided funds for a three year PhD study to be conducted in the field of radiotherapy at Herlev Hospital, Denmark.

Availability of data and materials

The datasets supporting the conclusions of this article are included within the article and its additional files.

\section{Authors' contributions}

MB carried out the prostate delineation, participated in designing the study, performed statistical analysis, carried out the sequence alignment and drafted the manuscript. CFB participated in the design of the study, revising the manuscript, and read and approved the final manuscript. All authors have read and approved the manuscript.

Authors' information

Claus F. Behrens, PhD, Honorary assoc. Prof., Herlev Hospital, Denmark.

Competing interests

The authors declare that they have no competing interests.

\section{Consent for publication}

Not applicable!

\section{Ethics approval and consent to participate}

The study was approved by the Danish national ethical committee, and voluntary informed consent was obtained for each participant according to the World Medical Association Declaration of Helsinki (1975/2000).

\section{Author details}

'Department of Oncology, Radiotherapy Research Unit, Herlev Hospital, University of Copenhagen, Herlev, Denmark. ${ }^{2}$ Center for Fast Ultrasound Imaging, Department of Electrical Engineering, Technical University of Denmark, DK-2800 Lyngby, Denmark. ${ }^{3}$ Center for Nuclear Technologies, Technical University of Denmark, DTU Risø Campus, Roskilde, Denmark.

Received: 24 September 2015 Accepted: 16 June 2016

Published online: 15 July 2016

\section{References}

1. Scarbrough TJ, Golden NM, Ting JY, Fuller CD, Wong A, Kupelian P a, et al. Comparison of ultrasound and implanted seed marker prostate localization methods: Implications for image-guided radiotherapy. Int J Radiat Oncol Biol Phys [Internet]. 2006 Jun 1 [cited 2012 Nov 27];65(2):378-87. Available from: http://www.ncbi.nlm.nih.gov/pubmed/16563658. 
2. Kupelian PA, Langen KM, Willoughby TR, Zeidan OA, Meeks SL. Image-Guided Radiotherapy for Localized Prostate Cancer: Treating a Moving Target. Semin Radiat Oncol. 2008;18(1):58-66.

3. Kitamura K. Three-dimensional intrafractional movement of prostate measured during real-time tumor-tracking radiotherapy in supine and prone treatment positions. Int J Radiat Oncol Biol Phys. 2002;53(5):1117-23.

4. Li HS, Chetty IJ, Enke CA, Foster RD, Willoughby TR, Kupellian PA, et al. Dosimetric consequences of intrafraction prostate motion. Int J Radiat Oncol Biol Phys [lnternet]. 2008 Jul 1 [cited 2015 Sep 2];71(3):801-12. Available from: http://www.ncbi.nlm.nih.gov/pubmed/18234439.

5. Dawson LA. A comparison of ventilatory prostate movement in four treatment positions. Int J Radiat Oncol Biol Phys. 2000;48(2).

6. Padhani A. Evaluating the effect of rectal distension and rectal movement on prostate gland position using cine MRI. Int J Radiat Oncol Biol Phys. 1999:44(3):525-33.

7. van Herk M. Quantification of organ motion during conformal radiotherapy of the prostate by three dimensional image registration. Int J Radiat Oncol Biol Phys. 1995;33(5):1311-20.

8. Tong $X$, Chen $X, L i J, X u$ Q, Lin M, Chen L, et al. Intrafractional prostate motion during external beam radiotherapy monitored by a real-time target localization system. J Appl Clin Med Phys. 2015;16(2):51-61.

9. Mayyas E, Chetty IJ, Chetvertkov M, Wen N, Neicu T, Nurushev T, et al. Evaluation of multiple image-based modalities for image-guided radiation therapy (IGRT) of prostate carcinoma: a prospective study. Med Phys [Internet]. 2013;40(4):041707. Available from: http://www.ncbi.nlm.nih.gov/pubmed/23556877.

10. Chan P, Dinniwell R, Haider M a, Cho Y-B, Jaffray D, Lockwood $G$, et al. Inter- and intrafractional tumor and organ movement in patients with cervical cancer undergoing radiotherapy: a cinematic-MRI point-of-interest study. Int J Radiat Oncol Biol Phys [Internet]. 2008 Apr 1 [cited 2013 Dec 30];70(5):1507-15. Available from: http://www.ncbi.nlm.nih.gov/pubmed/18164850.

11. Ghilezan MJ, Jaffray D a, Siewerdsen JH, Van Herk M, Shetty A, Sharpe MB, et al. Prostate gland motion assessed with cine-magnetic resonance imaging (cineMRI). Int J Radiat Oncol Biol Phys [Internet]. 2005 Jun 1 [cited 2012 Nov 1];62(2): 406-17. Available from: http://www.ncbi.nlm.nih.gov/pubmed/15890582.

12. Kron T, Thomas J, Fox C, Thompson A, Owen R, Herschtal A, et al. Intra-fraction prostate displacement in radiotherapy estimated from pre- and post-treatment imaging of patients with implanted fiducial markers. Radiother Oncol [Internet]. 2010 May [cited 2015 Sep 2];95(2): 191-7. Available from: http://www.ncbi.nlm.nih.gov/pubmed/20189667.

13. Adamson J, Wu Q. Inferences about prostate intrafraction motion from pre- and posttreatment volumetric imaging. Int J Radiat Oncol Biol Phys [Internet]. 2009 Sep 1 [cited 2015 Sep 2];75(1):260-7. Available from: http://www.pubmedcentral.nih.gov/articlerender. fcgi?artid=2730426\&tool=pmcentrez\&rendertype=abstract.

14. Lachaine M, Falco T. Intrafractional prostate motion management with the Clarity Autoscan system. Med Phys Int [Internet]. 2013;1(1):72-80. Available from: http://federation-fampo.org/web/uploads/MPI-2013-01.pdf\#page=72.

15. Bittner N, Butler WM, Reed JL, Murray BC, Kurko BS, Wallner KE, et al. Electromagnetic tracking of intrafraction prostate displacement in patients externally immobilized in the prone position. Int J Radiat Oncol Biol Phys [Internet]. 2010 Jun 1 [cited 2015 Sep 2];77(2):490-5. Available from: http://www.ncbi.nlm.nih.gov/pubmed/19775826.

16. Wilbert J, Baier K, Hermann C, Flentje M, Guckenberger M. Accuracy of real-time couch tracking during 3-dimensional conformal radiation therapy, intensity modulated radiation therapy, and volumetric modulated arc therapy for prostate cancer. Int I Radiat Oncol Biol Phys [Internet]. 2013 Jan 1 [cited 2015 Sep 2];85(1):237-42. Available from: http://www.ncbi.nlm.nih.gov/pubmed/22541958.

17. Curtis W, Khan M, Magnelli A, Stephans K, Tendulkar R, Xia P. Relationship of imaging frequency and planning margin to account for intrafraction prostate motion: analysis based on real-time monitoring data. Int J Radiat Oncol Biol Phys [Internet]. 2013 Mar 1 [cited 2015 Sep 2];85(3):700-6. Available from: http://www.ncbi.nlm.nih.gov/pubmed/22795802.

18. Langen KM, Willoughby TR, Meeks SL, Santhanam A, Cunningham A, Levine $L$, et al. Observations on real-time prostate gland motion using electromagnetic tracking. Int J Radiat Oncol Biol Phys [Internet]. 2008 Jul 15 [cited 2015 Sep 2];71(4):1084-90. Available from: http://www.ncbi.nlm.nih.gov/pubmed/18280057.

19. Li JS. Observations of prostate intrafractional motion during external beam radiation therapy. Ifmbe Proc. 2009;25(1):499-502.
20. Ballhausen H, Li M, Hegemann N-S, Ganswindt U, Belka C. Intra-fraction motion of the prostate is a random walk. Phys Med Biol [Internet]. 2015;60(2):549-63. Available from: http://stacks.iop.org/0031-9155/60/i=2/ $a=549$ ? key $=$ crossref. 1 d37a7c57f0aac3a6191 cff1 152ecde0.

21. Li JS, Lin M-H, Buyyounouski MK, Horwitz EM, Ma C-M. Reduction of prostate intrafractional motion from shortening the treatment time. Phys Med Biol [Internet]. 2013 Jul 21 [cited 2015 Sep 2];58(14):4921-32. Available from: http://www.pubmedcentral.nih.gov/articlerender. fcgi?artid=3940444\&tool=pmcentrez\&rendertype=abstract.

22. Mutanga TF, de Boer HCJ, Rajan V, Dirkx MLP, Incrocci L, Heijmen BJM. Day-to-day reproducibility of prostate intrafraction motion assessed by multiple $\mathrm{kV}$ and $\mathrm{MV}$ imaging of implanted markers during treatment. Int J Radiat Oncol Biol Phys [Internet]. 2012 May 1 [cited 2015 Sep 2];83(1): 400-7. Available from: http://www.ncbi.nlm.nih.gov/pubmed/22019244.

23. van der Meer S, Bloemen-van Gurp E, Hermans J, Voncken R, Heuvelmans D, Gubbels C, et al. Critical assessment of intramodality 3D ultrasound imaging for prostate IGRT compared to fiducial markers. Med Phys. 2013;40(7): 071707. Available from: http://scitation.aip.org/content/aapm/journal/ medphys/40/7/10.1118/1.4808359/, http://www.ncbi.nlm.nih.gov/pubmed/ 23822411.

24. Deegan T, Owen R, Holt T, Roberts L, Biggs J, McCarthy A, et al. Interobserver variability of radiation therapists aligning to fiducial markers for prostate radiation therapy. J Med Imaging Radiat Oncol [Internet]. 2013 Aug [cited 2015 Sep 2];57(4):519-23; quiz 524-5. Available from: http:// www.ncbi.nlm.nih.gov/pubmed/23870354.

25. Kotte ANTJ. Intrafraction motion of the prostate during external-beam radiation therapy: analysis of 427 patients with implanted fiducial markers. Int J Radiat Oncol Biol Phys. 2007;69(2).

26. Baker M, Behrens CF. Prostate displacement during transabdominal ultrasound image-guided radiotherapy assessed by real-time four-dimensional transperineal monitoring. Acta Oncol [Internet]. 2015 Oct [cited 2016 Jan 14];54(9):1508-14. Available from: http://www.ncbi.nlm.nih.gov/pubmed/26203927.

27. Abramowitz MC, Bossart E, Flook R, Wu X, Brooks R, Lachaine M, et al. Noninvasive Real-time Prostate Tracking Using a Transperineal Ultrasound Approach. Int J Radiat Oncol [lnternet]. 2012 Nov [cited 2015 May 10];84(3):S133. Available from: http//linkinghub.elsevier.com/retrieve/pii/S0360301612010899.

28. Choi Y, Kwak D-W, Lee H-S, Hur W-J, Cho W-Y, Sung GT, et al. Effect of rectal enema on intrafraction prostate movement during image-guided radiotherapy. J Med Imaging Radiat Oncol [Internet]. 2015;59(2):236-42. Available from: http://doi.wiley.com/10.1111/1754-9485.12239.

29. Vargas C, Saito Al, Hsi WC, Indelicato D, Falchook A, Zengm Q, et al. Cine-magnetic resonance imaging assessment of intrafraction motion for prostate cancer patients supine or prone with and without a rectal balloon. Am J Clin Oncol [Internet]. 2010 Feb [cited 2015 Sep 7];33(1):11-6. Available from: http://www.ncbi.nlm.nih.gov/pubmed/19730351.

30. Smeenk RJ, Louwe RJW, Langen KM, Shah AP, Kupelian PA, van Lin ENJT, et al. An endorectal balloon reduces intrafraction prostate motion during radiotherapy. Int J Radiat Oncol Biol Phys [Internet]. 2012 Jun 1 [cited 2015 Aug 17];83(2):661-9. Available from: http://www.ncbi.nlm.nih.gov/pubmed/ 22099035

31. Shelton J, Rossi PJ, Chen H, Liu Y, Master V a., Jani AB. Observations on prostate intrafraction motion and the effect of reduced treatment time using volumetric modulated arc therapy. Pract Radiat Oncol [Internet]. American Society for Radiation Oncology; 2011;1(4):243-50. Available from: http://dx.doi.org/10.1016/j.prro.2011.02.008.

32. Ng JA, Booth JT, Poulsen PR, Fledelius W, Worm ES, Eade T, et al. Kilovoltage intrafraction monitoring for prostate intensity modulated arc therapy: first clinical results. Int J Radiat Oncol Biol Phys [Internet]. 2012 Dec 1 [cited 2015 Sep 8];84(5):e655-61. Available from: http://www.ncbi.nlm.nih.gov/pubmed/22975613.

33. Lometti M. Intra-fraction prostate motion using MV fluoroscopy. Int J Radiat Oncol Biol Phys. 2005;63(2).

34. Wang KK, Vapiwala N, Deville C, et al. A study to quantify the effectiveness of daily endorectal balloon for prostate intrafraction motion management. Int J Radiat Oncol Biol Phys. 2012;83:1055-63. Available from: http://www. ncbi.nlm.nih.gov/pubmed/22115790. 